\title{
Numerical Approach to Processing of Biomechanical Experimental Results
}

\author{
Audrone Dumciene ${ }^{1}$, Saule Sipaviciene ${ }^{2}$ \\ ${ }^{I}$ Department of Health, Physical and Social Education, Lithuanian Sports University, \\ Sporto St. 6, LT-44221 Kaunas, Lithuania \\ ${ }^{2}$ Department of Applied Biology and Rehabilitation, Lithuanian Sports University, \\ Sporto St. 6, LT-44221 Kaunas, Lithuania \\ audrone.dumciene@lsu.lt
}

\begin{abstract}
The aim is to analyse the possibilities to approximate the data of biomechanical experiments using analytical functions, and to calculate parameters of human limb movement that are of interest to researchers, such as the maximum torque, motion duration, the time taken to achieve maximum torque, motion speed changes during the extension and flexion of limb. The Biodex 3 System Pro was used to collect the experimental data. Right leg extension motion parameters were measured 10 times. The experiment lasted three days, with 1-hour break to rest between tests. The foot extension parameters were: maximum torque $104.38 \pm$ $2.18 \mathrm{Nm}$, the time to peak torque $436 \pm 2.6 \mathrm{~ms}$, motion duration $1000 \pm 2.5 \mathrm{~ms}$. The torque values were measured using monitor. It was shown that it is appropriate to use the sixth-order polynomial to approximate the torque versus time curve. Polynomial coefficients with no less 16 significant digits should be used in calculations. Experimental and computational data are presented.
\end{abstract}

Index Terms-Measurement; curve fitting; approximation; error.

\section{INTRODUCTION}

The measurement system Multi-Joint System Biodex 3 Pro, designed for testing and rehabilitation of the human musculoskeletal system, can be used to analyse biomechanical motion parameters of human limb movement such as speed, torque, position, etc.

The system may be useful to test the knee, ankle, shoulder, elbow, forearm, and wrist motions. System operating principles and basic parameters are described in [1]-[4]. System reliability and validity have been studied in $[5],[6]$.

The measurement results are processed by specialized software and are visualized as it is more convenient for users in clinical practice and rehabilitation. However, such a reporting system is not always acceptable for researchers who need actual numerical values of certain parameters. For that purpose, the researchers have to assess the measurement results visually on the monitor screen. The errors of such evaluation are practically unavoidable due to the finite thickness of the curvature and line markers and researcher's eye position in respect to the measurement point and the

Manuscript received 3 November, 2015; accepted 22 August, 2016. investigator's experience. This procedure also takes a lot of time; the measurements must be repeated, which is not always possible.

The measuring curve continuity is also associated with the condition of investigating limb joints, muscles and nervous system. For the investigation of individual properties and due to the above-mentioned reasons, graphical characteristics of the observed processes may vary considerably [7]. Nonetheless, such charts describe the test object parameters indicating specific motions or processes. Therefore, the curves visualized on the screen can be used to evaluate the parameters of limb extension and flexion motions.

Study [6] results show that for some of the test cases, the difference between the results provided in the Biodex 3 report and the data taken from the files ("raw data") before they were processed with system software can be significant.

Therefore, additional means to have for "raw data" processing for researchers is important.

The purpose of the current study was to offer approximation equation customized for processing data received by the system Biodex Pro 3

Researchers are mostly interested in developed maximum torque $M_{\max }$, the time taken to achieve maximum torque $t_{\text {Mmax }}$, relaxation time $t_{r}$, curve slope inclination and a number of other parameters.

In experiments, the limb extension and flexion duration may vary from less than a second to a few seconds. Signal sampling frequency can be selected $2000 \mathrm{~Hz}$ or less, depending on the duration of movement studied by the researcher. In this way it is possible to obtain the necessary number of data samples. In order to calculate the motion parameters of interest to the investigator, the experimental data approximating the empirical function should be selected, or, in other words, mathematical model should be created. In general, the experimental data can be described as an unknown function

$$
y=f(x)+\varepsilon,
$$

where $x$-independent variable (in this case, the time or the rotation angle), $y$ - dependent variable (in this case, the rotation), $\varepsilon$ - error. As an experience of using the Biodex 3 
Pro shows, the approximating function may have more than one maximum [2]. Thus, for the calculation of the required parameter values, the researcher must choose the survey data approximating function and calculate the required parameter values based on this function.

A number of methods are used to approximate experimental data, including hierarchical nonlinear approximation [8], Wavelet Transform [9], approximation by piecewise cubic spline method [10], using linear and nonlinear regression [11], etc. But as it is indicated in [12], when this method is applied directly, the amount of required computer resources increases and the adjacent maximums can be approximated as a single extreme. In the study [13] the polynomial curve fitting method with varying powers was proposed, but the number of adapted parameters is larger than of traditional polynomials.

\section{Methods AND SAMPLES}

The approximation function can be written as

$$
y=(x, a, b, c, \ldots)
$$

where $x$ - independent variable; $a, b, c$ - function parameters.

The curve parameters evaluated from the experimental data in biomedical studies can be different in each individual test case. Therefore, the approximation function must be adjusted for each measurement and its parameters must be determined every time. These calculations must be performed in real time, because the limb extension and flexion motions may be repeated not just once during the same investigation. It is, therefore, appropriate fewer less computer resources to estimate its parameters.

It can be assumed that the distribution of measurement data of torque developed during human limb extension or flexion collected with Biodex 3 Pro, which could also be influenced by the measurement errors, corresponds to normal distribution.

Then the value of the function $y_{i}$, which corresponds to value $x_{i}$, can be considered as a value of a random quantity $Y_{i}$ with normal distribution

$$
N\left(f\left(x_{i}\right) ; \delta_{i}\right)
$$

where $f\left(x_{i}\right)$ - the value of an empirical function at the point $x_{i} ; \delta_{i}-$ the standard deviation, determined by the measurement errors.

It is possible that the measurement precision is equal at all measurement points $\left(\delta_{i}=\delta, i=1,2,3, \ldots, n\right)$.

The condition of maximum fitting of empirical function and measurement data can be written as

$$
\sum_{i=1}^{n}\left(y_{i}-f\left(x_{i}\right)\right)^{2}=\min .
$$

By using (4) it is possible to find parameters $a, b, c$ of approximation function $y=(x, a, b, c, \ldots)$.

Thus the condition can be written as

$$
\sum_{i=1}^{n}\left(y_{i}-f\left(x_{i}, a, b, c, \ldots\right)\right)^{2}=\min .
$$

Function (4) will reach its extreme when a certain function $E=E(a, b, c, \ldots)$ reaches a minimum.

This condition can be written as:

$$
\left\{\begin{array}{l}
\frac{\partial E}{\partial a}=0 \\
\frac{\partial E}{\partial b}=0 \\
\frac{\partial E}{\partial c}=0
\end{array}\right.
$$

Using (5) and (6) the equations can be formed:

$$
\begin{aligned}
& \sum_{i=1}^{n}\left(y_{i}-f\left(x_{i}, a, b, c, \ldots \cdot\left(\frac{\partial f}{\partial a}\right)_{x=x_{i}}=0\right.\right. \\
& \sum_{i=1}^{n}\left(y_{i}-f\left(x_{i}, a, b, c, \ldots \cdot\left(\frac{\partial f}{\partial b}\right)_{x=x_{i}}=0,\right.\right. \\
& \sum_{i=1}^{n}\left(y_{i}-f\left(x_{i}, a, b, c, \ldots \cdot\left(\frac{\partial f}{\partial c}\right)_{x=x_{i}}=0 .\right.\right.
\end{aligned}
$$

By solving (7)-(9) it is possible to calculate parameters $a$, $b, c, \ldots$ for $(5)$. For this purpose, it is required to know the exact expression of (5).

The typical torque versus time curve when extending the knee is shown in Fig. 1.

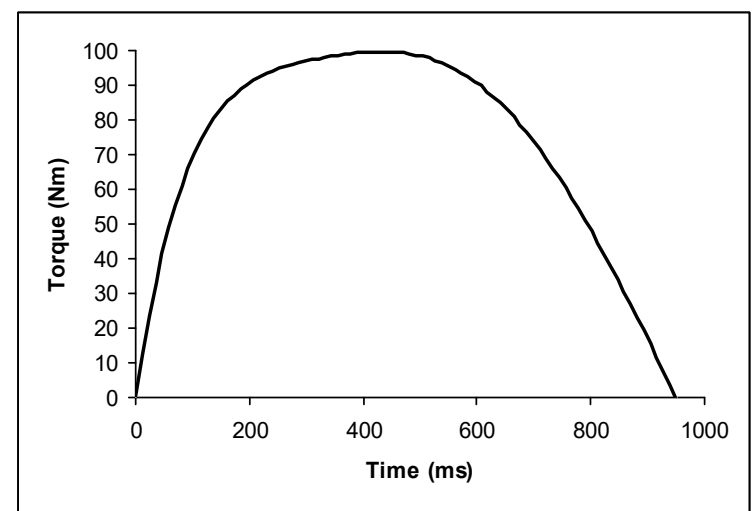

Fig. 1. Typical curve during knee extension.

If the function $f(x)$ there is a continuous area $D$; to locate the global maximum of this function in the area $D$, it is necessary to find points $x^{*}$ which are the points of function's global maximum in Euclidean space. So, we need to solve

$$
f\left(x^{*}\right)=\max \max f_{x \in D}(x) .
$$

The extreme condition

$$
\frac{\partial f(x)}{\partial x_{i}}=0
$$

where $i=1,2, \ldots, n$. The global maximum (10) can be found 
if the following condition is satisfied

$$
f\left(x^{*}\right) \geq f(x)
$$

for all $x$ in the interval $x \in D$.

To solve (10) it is possible to use the standard Math Lab function $\max (x)$.

The time from the start of a muscular contraction to the point of the highest torque development is called time to peak torque [3]. The time to peak torque is an indicator of the muscle's functional ability to produce torque quickly. So, when the maximum torque $M$ is found, the value $x^{*}$ corresponds to the time to peak torque.

Time Rate of Tension Development (TRTD) represented the amount of tension developed at the time of 0.2 seconds after start extension/flexion. By this time $80 \%-90 \%$ of peak torque should be achieved in the knee extensors [4]. TRTD can be calculated from (14), when $x=0.2 \mathrm{~s}$ and at any other time, by selecting the appropriate value of $x$.

Using (14), when conditions $x>0$ and $y=0$ are valid, we can calculate range of motion time $t_{\mathrm{md}}$. Derivative from (14) fulfilling the condition $\partial y / \partial x>0$ in the range from 0 to $x^{*}$ allows to calculate the speed of torque change in the range from $x=0$ to $x^{*}$. Derivative fulfilling the condition $\partial y / \partial x<0$ allows to calculate the speed of torque change in the range from $\mathrm{x}^{*}$ to $t_{\mathrm{md}}$. If in the range from $\mathrm{x}^{*}$ to $t_{\mathrm{md}}$ the condition $y^{\prime \prime}\left(x_{n+1}\right)-y^{\prime \prime}\left(x_{n}\right)<0$ is satisfied, this downslope of the curve is concave, indicating that there are difficulties with the development of strength.

The healthy male volunteer 22.6 years old, weight $82.6 \mathrm{~kg}$, height $191 \mathrm{~cm}$, was tested for experimental data collection. All experiments were performed according to one of the practices identified by Helsinki Declaration that is appropriate for Ethical Principles of experiments involving human being. The study protocol was approved by the Kaunas regional biomedical research ethics committee. Right leg extension motion parameters were measured 10 times using Biodex 3 System Pro. The experiment lasted three days with 1-hour break to rest between tests. The foot extension parameters were: the maximum torque $M_{\max }$ $104.38 \mathrm{Nm} \pm 2.18 \mathrm{Nm}$, the time to peak torque $t_{\mathrm{tp}} 436 \mathrm{~ms} \pm$ $2.6 \mathrm{~ms}$, motion duration $t_{\mathrm{md}} 1000 \mathrm{~ms} \pm 2.5 \mathrm{~ms}$, time rate of tension development $t_{0.2 \mathrm{~s}} 94,6 \mathrm{~ms} \pm 2.3 \mathrm{~ms}$.

During the first research phase, the torque values were measured using the monitor. This data was used to finding the most acceptable approximating analytic functions.

The signals of velocity, torque and position data in realtime were obtained from Biodex 3 Pro Analog Signal Access port output. A synchronizing pulse is issued whenever the real-time data are updated. So, using a digitized basic motion data you can process it and calculate data of interest to the investigator.

The example results of the knee extension torque measurement from the image displayed on the monitor screen are presented in Fig. 2

The n-th order polynomial can be used to approximate the experimental curve

$$
\begin{gathered}
y(x)=A_{0} x_{0}+A_{1} x+A_{2} x^{2}+A_{3} x^{3}+\ldots+ \\
+A_{n-2} x^{n-2}+A_{n-1} x^{n-1}+A_{n} x^{n},
\end{gathered}
$$

where $y(x)$ is the approximation function; $x_{0}=1$ and $A_{i}$ are polynomial coefficients.

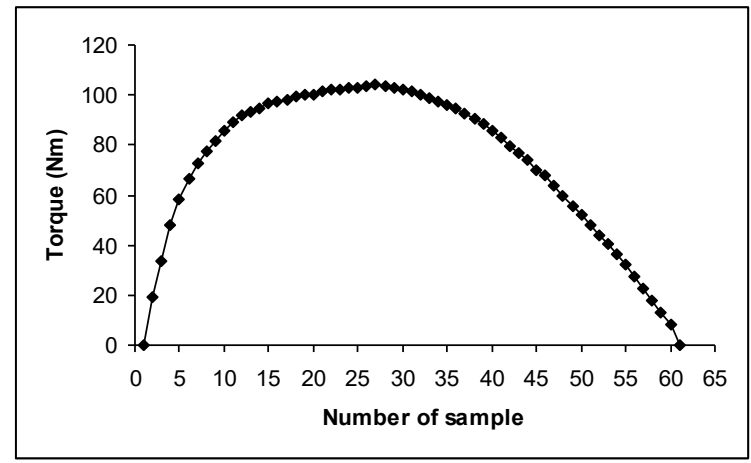

Fig. 2. The knee extension torque measurement results.

In order to have the best fit of approximation, it is required to select the best polynomial order and the number significant digits of polynomial coefficients for the approximation function.

The calculation results of curve fitting are presented in Table I.

TABLE I. THE SUM OF ERRORS SQUARES

\begin{tabular}{|c|c|}
\hline Polynomial order & Sum of error squares \\
\hline Second order & 3329,64 \\
\hline Third order & 1827,12 \\
\hline Fourth order & 836,76 \\
\hline Firth order & 282,83 \\
\hline Sixth order & 18,97 \\
\hline
\end{tabular}

On the basis of calculation results the sixth order polynomial was selected to approximate experimental results using the gradual approach method, by decreasing the sum of errors squares. The coefficient values of this polynomial are given in Table II and polynomial approximation (14).

TABLE II. COEFFICIENT VALUES OF THE SIXTH ORDER POLYNOMIAL.

\begin{tabular}{|c|c|}
\hline Coefficient & Coefficient value \\
\hline $\mathrm{A}_{0}$ & 0,0891911032406455 \\
\hline $\mathrm{A}_{1}$ & 1,20642545595392 \\
\hline $\mathrm{A}_{2}$ & $-6,36796833227302 \mathrm{E}-3$ \\
\hline $\mathrm{A}_{3}$ & $1,8097993503838 \mathrm{E}-5$ \\
\hline $\mathrm{A}_{4}$ & $-2,7645129296257 \mathrm{E}-8$ \\
\hline $\mathrm{A}_{5}$ & $2,08652574993833-\mathrm{E}-11$ \\
\hline $\mathrm{A}_{6}$ & $-6,15645573887369 \mathrm{E}-15$ \\
\hline
\end{tabular}

$$
\begin{gathered}
y(x)=A_{0}+A_{1} x+A_{2} x^{2}+A_{3} x^{3}+A_{4} x^{4}+ \\
+A_{5} x^{5}+A_{6} x^{6}
\end{gathered}
$$

The use of the polynomial of a higher order than sixth and the inclusion of more significant digits of the polynomial coefficients reduces the sum of error squares only slightly.

The distributions of errors between experimental data and results of calculation using fifth and sixth order polynomials are shown in Fig. 3 and Fig. 4 accordingly. 


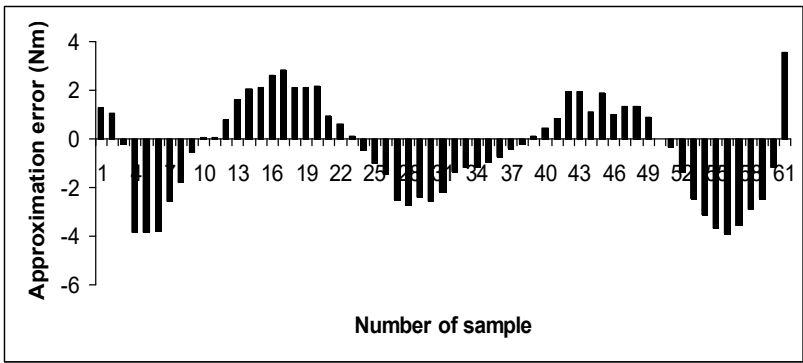

Fig. 3. Residual plot of errors after approximation by fifth order polynomial.

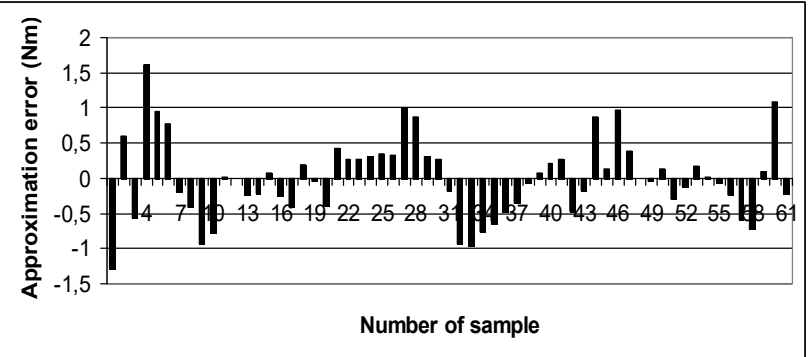

Fig. 4. Residual plot of errors after approximation by sixth order polynomial.

Distribution of relative errors using sixth order fitting polynomial, expressed in percentage, is shown in Fig. 5.

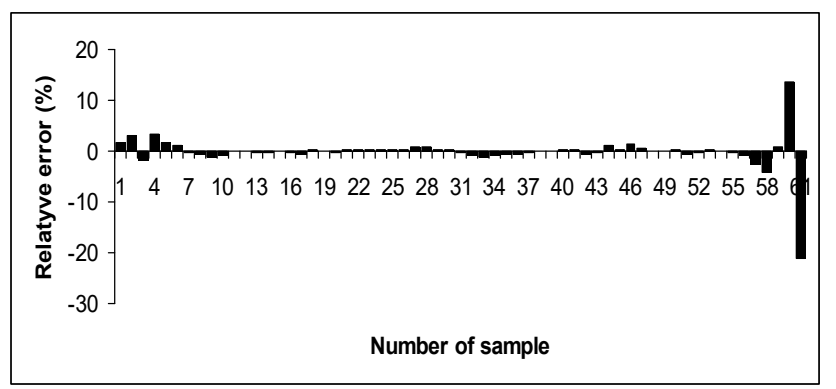

Fig. 5. Distribution of relative errors after approximation by sixth order polynomial.

The calculation results using (14): the maximum torque $M_{\max }=104.22 \mathrm{Nm}$ (relative error $1.06 \%$ ), time to peak torque $t_{\mathrm{pt}}=431.4 \mathrm{~ms}$ (relative error $0.15 \%$ ) and Time Rate of Tension Development $t_{0.2 \mathrm{~s}}=93.49 \mathrm{Nm}$ (relative error 1.77 $\%)$.

\section{CONCLUSIONS}

The sixth order polynomial in which the coefficients used with at least 16 significant digits for extension torque measurement results approximation it is appropriate to use. The relative error does not exceed $1.8 \%$ for calculating values for each of the three parameters $M_{\max }$, the $t_{\mathrm{op}}$ and $t_{0.2 \mathrm{~s}}$ using proposed approximation equation.

\section{REFERENCES}

[1] System 3 PRO. Application/operation manual. Biodex Medical Systems, Inc. Shirley, N.Y.

[2] Isokinetic Testing and Data Interpretation. Curve analysis. [Online]. Available: http://www.biodex.com/sites/default/files/manual-clinicalresources-data.pdf

[3] Isokinetic Testing and Data Interpretation. Data analysis. [Online]. Available: http://www.biodex.com/sites/default/files/manual-clinicalresources-data.pdf

[4] Biodex Multi-Joint System. Clinical Resource Manual. Isokinetic Source Book. [Online]. Available: http://mobilitysystems.se/edit/Klinisk-resurs-manual-fr-isokinetisktestning.pdf

[5] N. A. Taylor, R. H. Sanders, I. E. Howick, S. N. Stanley, "Static and dynamic assessment of the Biodex dynamometer", Eur J Appl Occup. Physiol, vol. 62, no. 3, pp. 180-188, 1991.

[6] J. Zawadzki, T. Bober, A. Siemienski, "Validity analysis of the Biodex System 3 dynamometer under static and isokinetic conditions", Acta of Bioengineering and Biomechanics, vol. 12, no. 4, pp. 25-32, 2010.

[7] P. Spencer-Wimpenny, Measurements. 2011. [Online]. Available: http://www.isokinetics.info/isokinetics/key-concepts/measurments. pdf?type $=$ raw

[8] D. Busby, Ch. L. Farmer, A. Iske, "Hierarchical nonlinear approximation for experimental design and statistical data fitting", SIAM Journal on Scientific Computing, vol. 29, no. 1, pp. 49-69, 2007. [Online]. Available: http://dx.doi.org/10.137/050639983

[9] P. S. Adison, The illustrated wavelet transform handbook, IOP Pub., 2002. [Online]. Available: http://dx.doi.org/10.1201/9781420033397

[10] M. Zamani, "A new, robust and applied model for approximation of huge data", Int. Journal of Advances in Applied Sciences (IJAAS 2013), vol. 2, no. 3, pp. 113-124, 2013.

[11] H. J. Motulsky, A. Christopoulos, Fitting models to biological data using linear and nonlinear regression. A practical guide to curve fitting. GraphPad Software Inc., San Diego CA, 2003.

[12] A. Chkifa, A. Cohen, G. Migliorati, F. Nobile, R. Tempone, "Discrete least squares polynomial approximation with random evaluations application to parametric and stochastic elliptic PDES", ESAIM: Mathematical Modelling and Numerical Anglysis, pp. 815-837, 2015. [Online]. Available: http://dx.doi.org/10.1051/m2an/2014050

[13] B. Cestisli, H. Kalkan, "Polynomial curve fitting with varying real powers", Elektronika ir Elektrotechnika, no. 6, pp. 117-122, 2011. [Online]. Available: http://dx.doi.org/10.5755/j01.eee.112.6.460 